\title{
Research on the Application of Chinese Traditional Pattern "Ruyi Pattern" in Buckle Design
}

\author{
Jun $\operatorname{Han}^{1}$ Yangyang Jiang ${ }^{1, *}{\mathrm{Jun} \mathrm{Li}^{1}}^{1}$ \\ ${ }^{1}$ School of Art and Design, Wuhan Institute of Technology, Wuhan, Hubei 430205, China \\ *Corresponding author. Email:1607547474@qq.com
}

\begin{abstract}
Ruyi pattern is an important symbol of traditional Chinese auspicious patterns. With the development of the times, Ruyi pattern has flexibility and versatility in the process of application and has become an inexhaustible source of humanistic elements in contemporary art design. This article takes Ruyi pattern as the research object, starting from the meaning, origin, development history and types of Ruyi pattern, to understand and analyze, refine and simplify the modeling characteristics of traditional Ruyi pattern. By combining the specific design practice of Ruyi pattern in the buckle, the application of traditional Ruyi pattern in art design and its aesthetic value in real life can be made clear.
\end{abstract}

Keywords: Ruyi pattern, Buckle design, Art design, Aesthetic value.

\section{INTRODUCTION}

Since the appearance of the Ruyi pattern, it has gone through the changes of the times. As one of the auspicious patterns, the Ruyi pattern is still popular today. The implied meaning of Ruyi pattern is auspicious and wishful, which has rich emotional connotation; it has a variety of shapes, beautiful and flexible lines, and it is beautiful, and it can be decorated with many kinds of utensils. The use and innovation of such a classic and longlasting Ruyi pattern is also one of the most favorite expression techniques of modern designers.

\section{THE MEANING AND ORIGIN OF RUYI PATTERN}

\subsection{The Meaning of Ruyi Pattern}

The allegorical characteristics of Chinese decorative art are formed in the background of traditional Chinese culture. In decorative art, the elements of decorative art such as patterns and patterns express the central theme of "auspiciousness", which is an eternal age. The longstanding theme deeply reflects China's traditional culture and embodies the humanistic spirit of Chinese traditional culture. Ruyi is famous for its unique form, which means everything goes well.
Therefore, the Ruyi patterns extracted from Ruyi also have beautiful meanings and symbols, and then materialized into people's daily life, playing the role of decoration and wishing on many utensils.

\subsection{The Origin of Ruyi Pattern}

From the perspective of the development and evolution of Ruyi pattern's modeling, it is itself derived from the fusion and evolution of a variety of cultural visual elements, absorbing the characteristics of Western ornamentation and the modeling characteristics of moire. The reason for its occurrence is closely related to the background of the times.

Ruyi originated from "claw fight", that is, itching, which is both beautiful and practical. Because of its clever and convenient use, it is called "Ruyi". As one of the eight treasures of Buddha, Ruyi has the function of recording scriptures and furnishings. Like the European Renaissance, the Wei-Jin era was also the era with the richest artistic spirit in China. In the Wei-Jin dynasties, Ruyi was popular for a time with the meaning of "auspicious and wishful ", and it was widely used. Ruyi's head also symbolized auspiciousness and wishful, which came into being, and became the best projection of the fusion of art in the Wei and Jin dynasties. 


\section{THE DEVELOPMENT HISTORY OF RUYI PATTERN}

The development of Ruyi pattern is mainly divided into the following three periods.

\subsection{Infancy}

The earliest development of Ruyi pattern can be traced back to primitive society and slave society. As the primary stage of human society development, the styles of artifacts are mostly simple and rough. The ancestors were full of awe and fantasy for the mysterious and unpredictable world. Therefore, the original totem has a huge influence on the production of auspicious patterns. The sprouting of the Ruyi pattern was in the important period of primitive society and slave society. The Majiayao spiral pattern and its deformed body, the cloud and thunder pattern of Shang and Zhou Dynasties, were the nascent stage of the Ruyi pattern.

\subsection{Development Period}

During the Qin-Han Dynasties, Buddhism was introduced to China, enriching the content of auspicious patterns and being widely used. From the Qin-Han Dynasties to the Wei-Jin and Southern and Northern Dynasties, the use of Ruyi became more and more common, and the Ruyi pattern entered the initial stage of development. "Curl Cao Ruyi Pattern" and "Auspicious Cloud Ruyi Pattern" are the most representative forms of Ruyi Pattern in the development period.

\subsection{Maturity}

From the Sui and Tang Dynasties to modern China, the society opened up, and the role of auspicious designs and decorations has been unprecedentedly developed. Ruyi pattern is developed in multiple ways on the basis of plant Ruyi pattern, combined with traditional auspicious ideas to express auspicious concept, and is widely used in the fields of architecture, furniture, clothing, ceramics, etc. And it is becoming mature, and Ruyi pattern has entered a highly popular period of development.

\section{TYPES OF RUYI PATTERN}

Ruyi pattern is extracted from the shape of the two ends of Ruyi ornaments, which can be connected with other things to form a new image, or it can be combined with various patterns to express different meanings. As a representative auspicious pattern, Ruyi pattern is loved by people and has become a common auspicious symbol. Ruyi pattern has a variety of expression methods and is highly decorative and adaptable.

Ruyi pattern is divided into the following four types:

\subsection{Heart-shaped Ruyi Pattern}

As shown in "Figure 1", the heart-shaped Ruyi pattern is formed by connecting two curved lines, with a concise and concise appearance, which summarizes the basic characteristics of the Ruyi pattern.

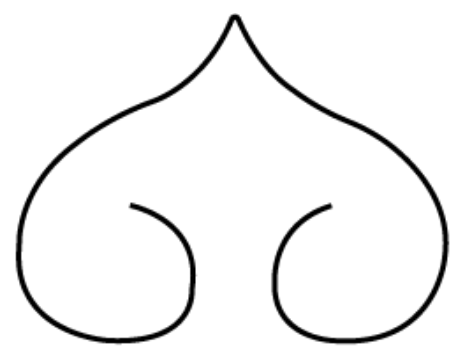

Figure 1 Heart-shaped Ruyi pattern.

\subsection{Curly Grass Ruyi Pattern}

The curly-grass Ruyi pattern is a combination of the curly-grass pattern and the Ruyi pattern. As shown in "Figure 2", the lines are smooth and curled. The beautiful meaning of the static Ruyi pattern is given to the dynamic curly grass pattern, which outlines a pattern image that is endless and full of romanticism.

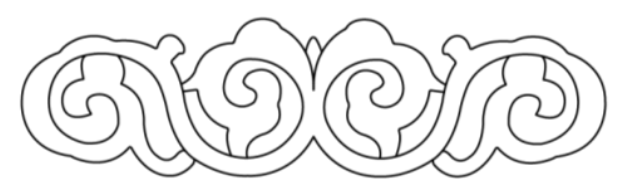

Figure 2 Curly Grass Ruyi Pattern.

\subsection{Ganoderma Ruyi Pattern}

Ganoderma Ruyi pattern is more common. The shape of Ganoderma lucidum itself is close to the shape of Ruyi pattern. The combination of Ganoderma and Ruyi symbolizes good luck. As shown in "Figure 3", they are combined into a composite pattern, which is a symbol of everything goes well. 


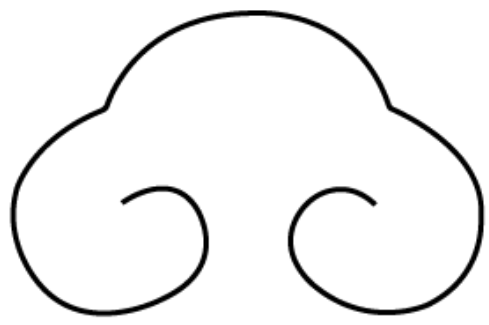

Figure 3 Ganoderma Ruyi Pattern.

\subsection{The Combination of Moire and Ruyi Pattern}

As shown in "Figure 4", the pattern is a combination of Moire and Ruyi pattern. It has the decorative characteristics of auspicious clouds and has the double meaning of "auspicious " and " wishful ", making it more meaningful.

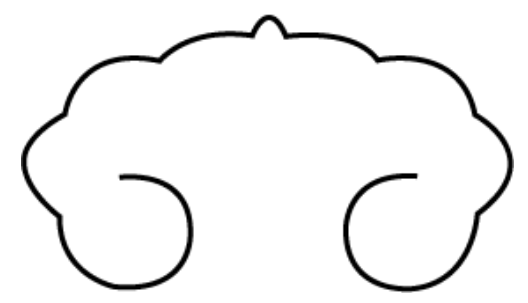

Figure 4 The combination of Moire and Ruyi pattern.

\section{APPLICATION OF RUYI PATTERN IN BUCKLE DESIGN}

\subsection{Extraction and Simplification of Ruyi Pattern}

Most of the Ruyi grains used in product design are transformed through deformation, as shown in "Figure 5". The deformation methods of Ruyi grain in product design include innovative transformation, generalization and refinement, and structural reorganization. The three-dimensional display of the Ruyi pattern in the buckle design requires attention to the necessary processing of the pattern, simplifying and emphasizing the overall characteristics of the Ruyi pattern, so that it conforms to the design style of the buckle. Therefore, the buckle has the meaning of beauty and good luck on the basis of practicality.

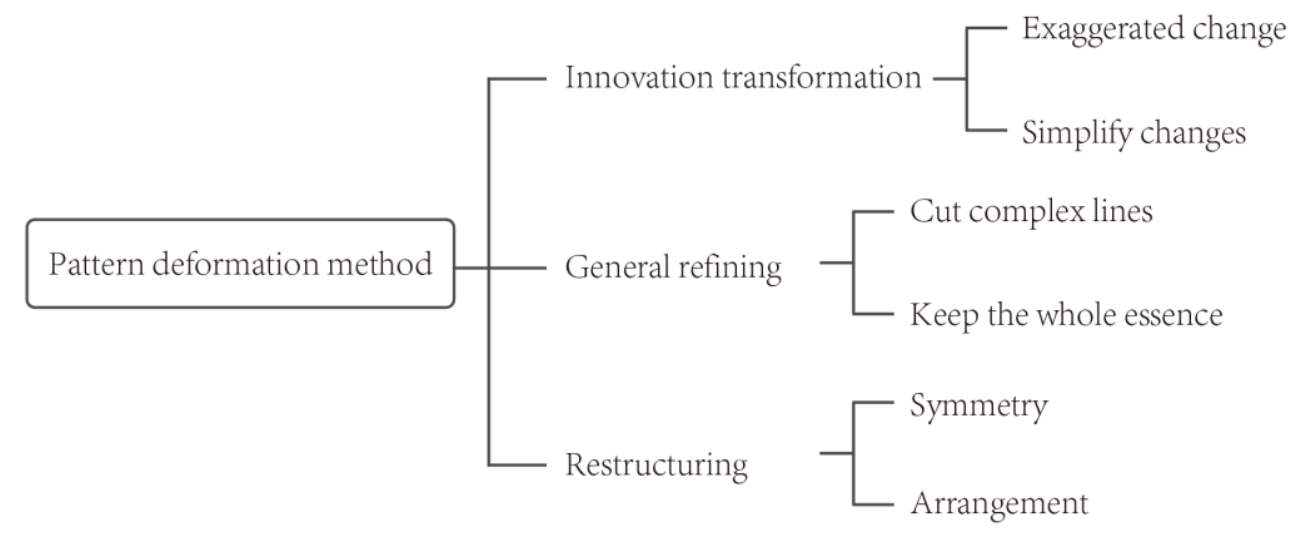

Figure 5 Pattern deformation method.

Modern design pursues simplicity but not simplicity. Therefore, in the process of using auspicious patterns, it is necessary to summarize, extract and simplify the overall characteristics of the object to achieve the purpose of expressing the meaning. "Figure 6" shows the extraction and simplification results of Ruyi pattern. Ruyi pattern has symmetrical and continuous composition characteristics. The refining process mainly includes exaggerated changes and simplified 
changes. This article chooses to simplify changes and omit the complicated and complicated Ruyi patterns, so that the extracted Ruyi pattern is concise and generous without losing its original rich connotation.

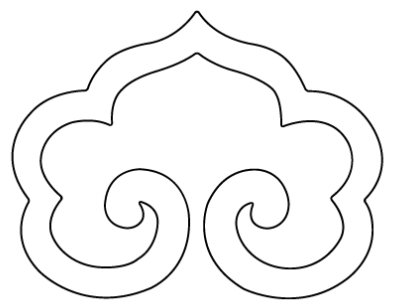

Figure 6 Extraction and Simplification of Ruyi pattern.

\subsection{The Specific Application of Ruyi Pattern in Buckle Design}

The buckle is composed of a male buckle and a female buckle. One end is fixed with a webbing, and the other end can be adjusted by a webbing. The length of the webbing can be adjusted according to different needs. The most common places to insert buckles are the seat belts of vehicles or amusement facilities, as well as the buckles on school bags, which generally play a fixed role.

Nowadays, the phenomenon of commodity homogeneity is becoming more and more serious. Most of the designs of buckles are simple geometric shapes, with the aid of rounded corners and monotonous color matching. As shown in "Figure. 7", the application of the Ruyi pattern in the design of the buckle can make the originally identical products appear in front of customers in a differentiated form. Ruyi pattern is indeed a kind of icing on the cake for the design of buckle. It has its unique meaning and entrusts people's desire for auspiciousness. The specific application of Ruyi pattern on the buckle will bring people to the good period of auspiciousness in life.
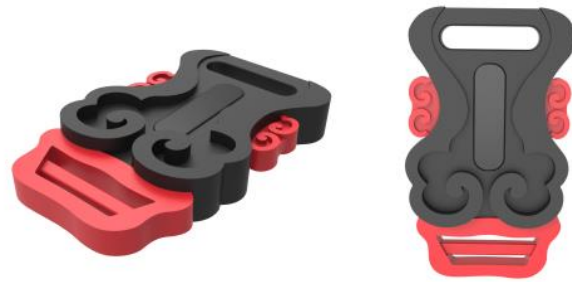

The buckle is redesigned by applying the Ruyi pattern, and the similarities between the Ruyi pattern and the shape of the buckle are reasonably extracted and reconstructed. The external form of the buckle is more spiritual and interesting, but also reflects the inheritance and innovation of Chinese traditional culture. In product design, the transformation of two-dimensional patterns into three-dimensional designs is an important direction. The traditional auspicious patterns not only play a decorative role in product design, but also reflect rich cultural connotations and humanistic spirit. The Ruyi pattern realizes the three-dimensional expression of traditional decorative patterns through the design process and methods of induction, refinement, reorganization, and simplification, so that the modern buckle design has more traditional Chinese cultural heritage.

\section{CONCLUSION}

With the rapid development of the global economy today, design and economic development are inseparable. How to inherit, develop and promote traditional Chinese decorative patterns, draw nourishment from the extensive and profound Chinese traditional culture, improve design connotation, and enhance design taste is our common concern. As a traditional Chinese auspicious pattern, the Ruyi pattern has profound meaning and beautiful shape. It has been continuously enriched and improved, and developed in diversification. The application of the Ruyi pattern in the design of the buckle makes full use of its styling elements and its characteristics. The design of the buckle shows the cultural connotation contained in the Ruyi pattern and the charm of traditional Chinese culture. In the specific design practice, the fit point between the Ruyi pattern and the buckle design can be found. The simplified Ruyi pattern makes the buckle delicate and durable, and the decorative effect is obvious. The buckle design presents a unique personality and artistic style.

\section{AUTHORS' CONTRIBUTIONS}

This article is completed by Jun Han, Yangyang Jiang, and Jun Li. Jun Han offered theoretical guidance for this paper, Yangyang Jiang is the main writer of this paper, and Jun Li helped to gather the references.

Figure 7 Buckle design. 


\section{REFERENCES}

[1] Huang Bingxiang. Research on the contemporary application of Chinese traditional Ruyi patterns[J]. Western Leather, 2020, 42(01):118.

[2] Zhong Yong. The mathematical analysis and design application of Ruyiwen[J]. Yihai, 2020(06):94-95.

[3] Tai Xuechun. On the tracing, development and application of the traditional Chinese pattern "Ruyiwen"[J]. Art Technology, 2019, 32(10): $148+155$.

[4] Liu Xiaoli. Talking about the Ruyi Pattern in Chinese Traditional Auspicious Patterns[J]. Packaging World, 2011(02): 79-81.

[5] Hao Ming. Research on the artistic symbols of Ruyiwen in the Wei, Jin, Southern and Northern Dynasties[D]. Hunan University of Technology, 2010.

[6] Wang Qunchao. The application of traditional Chinese auspicious patterns in the design of new Chinese furniture [D]. Tianjin University of Science and Technology, 2017.

[7] $\mathrm{Li} \mathrm{Na}$. Inheritance of Chinese traditional auspicious patterns and its influence on modern design[D]. Qingdao University of Science and Technology, 2011.

[8] Liu Li. The evolution of Chinese auspicious patterns and the exploration of its cultural connotation[D]. Jilin Academy of Arts, 2007.

[9] Zhu Junjun. Persimmon (everything) wishful thinking-Yuhang auspicious cultural product design research [D]. China Academy of Art, 2010.

[10] Cao Jinfeng. Research on the Ruyi Cloud Head of Women's Tops in the Late Qing Dynasty and the Early Republic of China [D]. Beijing Institute of Fashion Technology, 2016.

[11] Zeng Jia. The influence of Chinese traditional auspicious patterns on art design[J]. Design Art Research, 2012, 2(03): 22-26.

[12] Fu Xi. Application and Research of Ruyi Moire Pattern in Scarf Creative Design [D]. Hunan Normal University, 2016.

[13] Liu Qian. Research on the symbolic meaning of traditional auspicious patterns and their influence on modern design [D]. Hunan Normal University, 2014.

[14] Chen Zhiquan. Research on the application of auspicious patterns in logo design[J]. China Packaging Industry, 2015(18): 47. 